\title{
On zero-sum free subsets of length 7
}

\author{
Pingzhi Yuan* \\ School of Mathematics, South China Normal University \\ Guangzhou 510631, P.R.China \\ mcsypz@mail.sysu.edu.cn \\ Xiangneng Zeng \\ Department of Mathematics, Sun Yat-Sen University \\ Guangzhou 510275, P.R.China
}

Submitted: Nov 2, 2009; Accepted: Jul 26, 2010; Published: Aug 9, 2010

Mathematics Subject Classifications: primary 11B75; secondary 11B50

\begin{abstract}
Let $G$ be a finite additively written abelian group, and let $X$ be a subset of 7 elements in $G$. We show that if $X$ contains no nonempty subset with sum zero, then the number of the elements which can be expressed as the sum over a nonempty subsequence of $X$ is at least 24 .
\end{abstract}

\section{Introduction}

Let $G$ be an additive abelian group and $X \subseteq G$ a subset of $G$. We denote by $f(G, X)=$ $f(X)$ the number of nonzero group elements which can be expressed as a sum of a nonempty subset of $X$. For a positive integer $k \in \mathbb{N}$, let $f(k)$ denote the minimum of all $f(G, X)$, where the minimum is taken over all finite abelian groups $G$ and all zero-sum free subsets $X \subset G$ with $|X|=k$. The invariant $f(k)$ was first studied by R. B. Eggleton and P. Erdős in 1972 [1]. For every $k \in \mathbb{N}$ they obtained a subset $X$ in a cyclic group $G$ with $|X|=k$ such that

$$
f(k) \leqslant f(G, X)=\left\lfloor\frac{1}{2} k^{2}\right\rfloor+1 .
$$

And J. E. Olson [2] proved that

$$
f(k) \geqslant \frac{1}{9} k^{2} .
$$

Moreover, Eggleton and Erdős determined $f(k)$ for all $k \leqslant 5$, and they stated the following conjecture (which holds true for $k \leqslant 5$ ):

\footnotetext{
* Supported by NSF of China (No. 10971072) and by the Guangdong Provincial Natural Science Foundation (No. 8151027501000114).
} 
Conjecture 1.1. For every $k \in \mathbb{N}$ there is a cyclic group $G$ and a zero-sum free subset $X \subset G$ with $|X|=k$ such that $f(k)=f(G, X)$.

Recently, Weidong Gao et al. [3] proved that $f(6)=19$ and G.Bhowmik et al. [5] showed that $f(G, X) \geqslant 24$ (the lower bound is sharp), where $G$ is a cyclic group, $|X|=7$. Together with the conjecture above, we have that $f(7)=24$. The main aim of the present paper is to show the following theorem.

Theorem 1.1. $f(7)=24$.

In Section 2, we fix the notation. Sections 3 and 4 are devoted to the tools and lemmas needed in the proof of Theorem 1.1. In Section 5, we prove Theorem 1.1 with the help of a $\mathrm{C}++$ program.

Throughout this paper, let $G$ denote an additive finite abelian group.

\section{Notation}

We follow the conventions of [6] and [3] for notation concerning sequences over an abelian group.

We denote by $\mathbb{N}$ the set of positive integers, and $\mathbb{N}_{0}=\mathbb{N} \cup\{0\}$. For real numbers $a, b \in \mathbb{R}$ we set $[a, b]=\{x \in \mathbb{Z} \mid a \leqslant x \leqslant b\}$.

Let $\mathcal{F}(G)$ denote the multiplicative, free abelian monoid with basis $G$. The elements of $\mathcal{F}(G)$ are called sequences over $G$. An element $X \in \mathcal{F}(G)$ will be written in the form

$$
X=g_{1} \cdot \ldots \cdot g_{l}=\prod_{g \in G} g^{v_{g}(X)}
$$

where $v_{g}(X) \in \mathbb{N}_{0}$ is the multiplicity of $g$ in $X$. For a sequence $X$ above we have:

$$
\begin{gathered}
|X|=l=\sum_{g \in G} v_{g}(X) \in \mathbb{N}_{0} \text { the length of } X, \\
\sigma(X)=\sum_{i=1}^{l} g_{i}=\sum_{g \in G} v_{g}(X) g \in G \text { the sum of } X, \\
\sum(X)=\left\{\sum_{i \in I} g_{i} \mid \emptyset \neq I \subset[1, l]\right\} \quad \text { the set of subsums of } X .
\end{gathered}
$$

We say that $X$ is

- zero-sum free if $0 \notin \sum(X)$,

- a zero-sum sequence if $\sigma(X)=0$,

- squarefree if $v_{g}(X) \leqslant 1$ for all $g \in G$, moreover, a squarefree sequence can be considered as a subset of $G$. 
For a zero-sum free sequence $X$ over $G$, we have:

$$
\begin{gathered}
f(G, X)=f(X)=\left|\sum(X)\right|, \\
f(G, k)=\min \{f(X) \mid X \in \mathcal{F}(G) \text { zero-sum free, squarefree and }|X|=k\}
\end{gathered}
$$

and set $f(G, k)=\infty$ when there are no sequences in $G$ of the above form.

$$
f(k)=\min \{f(G, k) \mid G \text { run over all finite abelian groups }\}
$$

- Let $D(G)$ denote the Davenport's constant of $G$ and $r(G)$ the rank of $G$.

- Let ol $(G)$ denote the maximal length of a sequence $X$ over $G$ which is zero-sum free and squarefree. The invariant $o l(G)$ is called the Olson constant of $G$.

\section{Preliminaries}

Lemma 3.1. 1. If $k \in \mathbb{N}$ and $X=X_{1} \cdot \ldots \cdot X_{k} \in \mathcal{F}(G)$ is a zero-sum free sequence, then

$$
f(X) \geqslant f\left(X_{1}\right)+\cdots+f\left(X_{k}\right) .
$$

2. If $X \subset G$ is zero-sum free, $|X|=k$ and $k \in \mathbb{N}$, then

$$
f(X) \begin{cases}=1, & \text { if } k=1 \\ =3, & \text { if } k=2 \\ \geqslant 5, & \text { if } k=3 \\ \geqslant 6, & \text { if } k=3 \text { and } 2 g \neq 0 \text { for all } g \in X \\ \geqslant 2 k, & \text { if } k \geqslant 4 .\end{cases}
$$

Proof. 1. See [6] Theorem 5.3.1.

2. See [6] Corollary 5.3.4.

Lemma 3.2. $([3]) f(5)=13, f(6)=19$.

Lemma 3.3. ([5]) $f(G, 7) \geqslant 24$, where $G$ is a cyclic group. Furthermore, let $G=C_{25}$ and $X=\{5,10,1,6,11,16,21\}$, then $f(X)=24$.

Lemma 3.4. Let $X \subset G$ be a zero-sum free subset of $G$ and $|X|=7$. If $X$ contains an element of order 2 , then $f(X) \geqslant 25$.

Proof. See [3] Theorem 3.2. 


\section{Some bounds on subset $S$}

The lemmas in this section follows mainly from A. Pixton [7].

Lemma 4.1. ([7] Lemma 4.3) Let $G$ be a finite abelian group and let $X \subseteq G \backslash\{0\}$ be a generating set for $G$. Suppose $S$ is a nonempty proper subset of $G$, then

$$
\sum_{x \in X}|(S+x) \backslash S| \geqslant|X|
$$

Lemma 4.2. ([7] Lemma 4.4) Let $G$ be a finite abelian group and let $X \subseteq G \backslash\{0\}$ be a generating set for $G$. Suppose $f: G \rightarrow \mathbb{Z}$ is a function on $G$. Then

$$
\sum_{\substack{x \in X \\ g \in G}} \max \{f(g+x)-f(g), 0\} \geqslant(\max (f)-\min (f))|X| .
$$

The proofs of the following two Lemmas are essential from A. Pixton ([7] Theorem 4.5). For the convenience of the reader, we present the proof here.

Lemma 4.3. Let $G$ be a finite abelian group and let $X \subseteq G \backslash\{0\}$ be a generating set for $G$. Suppose $S \subseteq G$ satisfies $|(S+x) \backslash S| \leqslant m$, for $m \in \mathbb{N}$ and all $x \in X$, and for $Y \subset X$ and $H=<Y>\nsubseteq G$, define a function $f: G / H \rightarrow \mathbb{Z}$ by $f(a)=|(a+H) \cap S|$ for $a \in G / H$. Then

$$
\max (f)-\min (f) \leqslant m \text {. }
$$

Proof. First, without loss of generality, we may replace $Y$ by a minimal subset of $Y$ that still generates $H$, and we still denote it by $Y$. Then we may replace $X$ by a minimal subset $X_{0}$ of $X$ that satisfies $Y \subset X_{0} \subset X$ and $\left\langle X_{0}\right\rangle=G$. For the convenience, we still label it by $X$. Also, if $|H| \leqslant m$, the result is trivial. Since

$$
\begin{aligned}
|(S+x) \backslash S| & =|(S-x) \backslash S| \\
& =\sum_{a \in G / H}|((S-x) \backslash S) \cap(a+H)| \\
& =\sum_{a \in G / H}|(S-x) \cap(a+H)|-|(S-x) \cap S \cap(a+H)| \\
& =\sum_{a \in G / H}|S \cap(a+x+H)|-|(S-x) \cap S \cap(a+H)| \\
& \geqslant \sum_{a \in G / H} \max \{f(a+x)-f(a), 0\}
\end{aligned}
$$

It follows that

$$
\begin{aligned}
m|X \backslash Y| & \geqslant \sum_{x \in X \backslash Y}|(S+x) \backslash S| \\
& \geqslant \sum_{x \in X \backslash Y} \sum_{a \in G / H} \max \{f(a+x)-f(a), 0\} \\
& \geqslant(\max (f)-\min (f))|X \backslash Y|
\end{aligned}
$$


by Lemma 4.2. Since $H \nsubseteq G,|X \backslash Y|>0$, then the result follows immediately from $(2)$.

Lemma 4.4. Let $G$ be a finite abelian group, $X \subseteq G \backslash\{0\}$ a generating set for $G, Y \subset X$ and $H=<Y>\nsubseteq \mathbb{G}$. Suppose $|H|>m$ and $|G / H|>m$, where $m \in \mathbb{N}$, and suppose $S \subseteq G$ satisfies $|(S+x) \backslash S| \leqslant m$ for all $x \in X$. Then

$$
\min \{|S|,|G \backslash S|\} \leqslant m^{2} .
$$

Proof. Define a function $f: G / H \rightarrow \mathbb{Z}$ by $f(a)=|(a+H) \cap S|$ for $a \in G / H$. We have

$$
\max (f)-\min (f) \leqslant m
$$

by Lemma 4.3 . Then by replacing $S$ by $G / S$ if necessary, we can assume that $f(a) \neq|H|$ for any $a \in G / H$. The reason is that

$$
|(G \backslash S+x) \backslash(G \backslash S)|=|(S+x) \backslash S|
$$

Thus we can apply Lemma 4.1 to obtain that

$$
\begin{aligned}
m|Y| & \geqslant \sum_{x \in Y}|(S+x) \backslash S| \\
& =\sum_{a \in G / H} \sum_{x \in Y}|(S \cap(a+H)+x) \backslash(S \cap(a+H))| \\
& \geqslant|\operatorname{supp}(f)||Y|
\end{aligned}
$$

where $\operatorname{supp}(f)=\{a \in G / H \mid f(a) \neq 0\}$ is the support of $f$. Since $|G / H|>m$, this implies that $f(a)=0$ for some $a$, and thus $f(a) \leqslant m$ for all $a \in G / H$. Then $|S|=\sum_{a \in G / H} f(a) \leqslant$ $\max (f)|\operatorname{supp}(f)| \leqslant m^{2}$, as desired.

Lemma 4.5. ([7] Theorem 5.3) Let $G$ be a finite abelian group of rank greater than 2 and let $X \subset G \backslash\{0\}$ be a generating set for $G$ consisting only of elements of order greater than 2. Suppose $S \subset G$ satisfies $|(S+x) \backslash S| \leqslant 3$ for all $x \in X$. Then $\min \{|S|,|G \backslash S|\} \leqslant 5$.

Lemma 4.6. Let $G$ be a finite abelian group of rank greater than 2 , and $X \subseteq G$ a zero-sum free generating set consisting of only elements of order greater than 2 , and let $S=\sum(X)$. Suppose that $|X| \geqslant 5$ and $|G| \geqslant 29$, then $|S| \geqslant 24$, or $|S| \geqslant 4|X|-3$, or there is some $x \in X$ satisfies $\langle X \backslash\{x\}\rangle=G$ and $|S|-\left|\sum(X \backslash\{x\})\right| \geqslant 4$.

Proof. If there is an element $x \in X$ such that $\langle X \backslash\{x\}\rangle \neq G$, then

$$
S=\sum(X \backslash\{x\}) \uplus\{x\} \uplus \sum(X \backslash\{x\})+x
$$

is a disjoint union. It follows that $|S|=2\left|\sum(X \backslash\{x\})\right|+1 \geqslant 2 \times 2(|X|-1)+1=4|X|-3$ by Lemma 3.1. Hence we may assume that $\langle X \backslash\{x\}\rangle=G$ for all $x \in X$. 
have

Now if $|S|-\left|\sum(X \backslash\{x\})\right| \leqslant 3$ for all $x \in X$, since $\sum(X \backslash\{x\}) \subset(S-x) \cap S$, then we

$$
\begin{aligned}
|(S-x) \backslash S| & =|S-x|-|(S-x) \cap S| \\
& \leqslant|S|-\left|\sum(X \backslash\{x\})\right| \\
& \leqslant 3 .
\end{aligned}
$$

It follows from Lemma 4.5 that $\min \{|S|,|G \backslash S|\} \leqslant 5$. Notice that $|S| \geqslant 2|X| \geqslant 10$, then $|G \backslash S| \leqslant 5$ and $|S| \geqslant|G|-5 \geqslant 24$, as desired.

\section{Other lemmas before the proof}

In this section, we present some Lemmas that will be used in the proof of the main result.

Lemma 5.1. Let $X \subseteq G$ be a zero-sum free generating set of $G,|X|=4$, and $X$ has no element of order 2. If $r(G) \geqslant 3$ and $G \neq C_{2} \oplus C_{2} \oplus C_{4}$, then $f(X) \geqslant 12$.

Proof. Let $X=x_{1} \cdot x_{2} \cdot x_{3} \cdot x_{4}$. If there are distinct indices $i, j, k \subset[1,4]$ such that $x_{i}=x_{j}+x_{k}$, without loss of generality, we may assume that $x_{1}=x_{2}+x_{3}$. Since $r(G) \geqslant 3$, then $x_{4} \notin\left\langle x_{1}, x_{2}, x_{3}\right\rangle$, and so

$$
\sum(X)=\sum\left(x_{1} x_{2} x_{3}\right) \biguplus\left\{x_{4}\right\} \biguplus\left(x_{4}+\sum\left(x_{1} x_{2} x_{3}\right)\right)
$$

is a disjoint union. It follows from Lemma 3.1 that $f(X) \geqslant 2 f\left(x_{1} x_{2} x_{3}\right)+1 \geqslant 13$.

Now we consider the case that $x_{i} \neq x_{j}+x_{k}$ for all distinct indices $i, j, k \in[1,4]$. If there is no index $i \in[1,4]$ such that $x_{i}=\sum_{j \neq i} x_{j}$, then $x_{1}, x_{2}, x_{3}, x_{4}, x_{1}+x_{2}, x_{1}+x_{3}, x_{1}+$ $x_{4}, x_{1}+x_{2}+x_{3}, x_{1}+x_{2}+x_{4}, x_{1}+x_{3}+x_{4}, x_{2}+x_{3}+x_{4}, x_{1}+x_{2}+x_{3}+x_{4}$ are pairwise distinct, so $f(X) \geqslant 12$.

Otherwise, we can assume $x_{1}=x_{2}+x_{3}+x_{4}$.

If $x_{\tau(1)}+x_{\tau(2)}=x_{\tau(3)}+x_{\tau(4)}$, where $\tau$ is an element of the symmetric group on $[1,4]$, then the two equations imply that there is some $x_{i}$ of order 2 , a contradiction.

If there is an index $i \in[1,4]$ such that $x_{i} \neq \sum_{j \neq i} x_{j}$, say, $x_{4} \neq x_{1}+x_{2}+x_{3}$, then $x_{1}, x_{2}, x_{3}, x_{4}, x_{1}+x_{2}+x_{3}, x_{1}+x_{2}, x_{1}+x_{3}, x_{1}+x_{4}, x_{2}+x_{3}, x_{2}+x_{4}, x_{3}+x_{4}, x_{1}+x_{2}+x_{3}+x_{4}$ are pairwise distinct, so $f(X) \geqslant 12$.

If $x_{i}=\sum_{j \neq i} x_{j}$ for all indices $i \in[1,4]$, then the 4 equations imply $4 x_{4}=0, x_{1}=$ $x_{4}+g_{1}, x_{2}=x_{4}+g_{2}$ and $x_{3}=-x_{4}+g_{1}+g_{2}$, where $g_{1}, g_{2}$ is of order 2 . It follows that $G=\langle X\rangle \cong C_{2} \oplus C_{2} \oplus C_{4}$, again a contradiction. We are done.

Lemma 5.2. Let $C_{n}$ be a cyclic group of order $n, S \subset C_{n}$ a subset of $G$. Suppose that d is a generator of $C_{n}$ and $x \in C_{n}$ is an element of order greater than 2. Then we have:

1. $|(S+x) \backslash S|=|(S-x) \backslash S|$.

2. If $S$ is an arithmetic progression of difference $d$, then

$$
|(S+x) \backslash S|=\min \{|S|, n-|S|, k, n-k\},
$$


where $k \in[1, n-1]$ is the integer with $x=k d$.

3. If $S=S_{1} \uplus S_{2}$ is a disjoint union, where $S_{1}, S_{2}$ are arithmetic progressions of difference $d$ and $S$ is not an arithmetic progressions of difference d. Suppose that $2 \leqslant$ $|S| \leqslant n-2$, then $|(S+x) \backslash S| \geqslant 1$.

4. Let $S$ be as in 3, and moreover $5 \leqslant|S| \leqslant n-5$ and $n=2 r, r$ is a positive integer, then $|(S+x) \backslash S| \geqslant 2$. Furthermore the equality holds only when $x$ is one of the following cases:

(a): $x= \pm d$.

(b): $x= \pm 2 d$. In the case, $S=\{g, g+d, \ldots, g+(t-1) d, g+t d\} \uplus\{g+(t+2) d\}$ or $S=\{g\} \uplus\{g+2 d, g+3 d, \ldots, g+(t-1) d, g+t d\}, g \in G$ and $t \in[3, n-5]$.

(c): $x= \pm(r-1) d$. In the case, ||$S_{1}|-| S_{2}|| \leqslant 2$.

5. If $S=S_{1} \cup S_{2} \cup S_{3}$ is a disjoint union of 3 arithmetic progressions of difference $d$, $x= \pm 3 d$, and $8 \leqslant|S| \leqslant n-7$, then $|(S+x) \backslash S| \geqslant 2$.

Proof. 1. It is obvious.

2. Obviously.

3. For a counterexample, we may assume that $S_{1}=\left\{g_{1}, g_{1}+d, \ldots, g_{1}+t_{1} d\right\}, S_{2}=$ $\left\{g_{2}, g_{2}+d, \ldots, g_{2}+t_{2} d\right\}$ and $|(S+x) \backslash S|=0$. The proof is divided into the following two cases:

Case 3.1: If $g_{1}+x \in S_{1}$, then there is an integer $k \in\left[0, t_{1}-1\right]$ such that $g_{1}+k d+x=$ $g_{1}+t_{1} d$, however $g_{1}+(k+1) d \in S_{1}$ and $g_{1}+(k+1) d+x \notin S$ yield a contradiction. The proof of the case $g_{2}+x \in S_{2}$ is similar.

Case 3.2: If $g_{1}+x \in S_{2}$ and $g_{2}+x \in S_{1}$, then $S_{1}+x \subseteq S_{2}$ and $S_{2}+x \subseteq S_{1}$. Hence $\left|S_{1}\right|=\left|S_{1}+x\right| \leqslant\left|S_{2}\right|$ and similarly $\left|S_{2}\right| \leqslant\left|S_{1}\right|$. It follows that $\left|S_{1}\right|=\left|S_{2}\right|, g_{1}+x=g_{2}$ and $g_{2}+x=g_{1}$, and hence $g_{1}=g_{2}+x=g_{1}+x+x$ and $2 x=0$, again a contradiction. We are done.

4. Without loss of generality, we may assume $\left|S_{1}\right| \geqslant\left|S_{2}\right|$. Let $r=\frac{n}{2}, S_{1}=\left\{g_{1}, g_{1}+\right.$ $\left.d, \ldots, g_{1}+t_{1} d\right\}, S_{2}=\left\{g_{2}, g_{2}+d, \ldots, g_{2}+t_{2} d\right\}, U_{1}=\left\{g_{1}+\left(t_{1}+1\right) d, g_{1}+\left(t_{1}+2\right) d, \ldots, g_{2}-d\right\}$ and $U_{2}=\left\{g_{2}+\left(t_{2}+1\right) d, g_{2}+\left(t_{2}+2\right) d, \ldots, g_{1}-d\right\}$. If $x= \pm d$ then $|(S+x) \backslash S|=2$. Since $|(S+x) \backslash S|=|(S-x) \backslash S|, n=2 r$ and $x$ is an element of order greater than 2 , then without loss of generality, we may assume that $x=k d, k \in[2, r-1]$.

Case 4.1: $k \in[3, r-2]$.

Subcase 4.1.1: If $\left|S_{1}\right| \geqslant k$, then $\left|U_{1}\right|+\left|U_{2}\right|=n-|S| \geqslant 5$ implies that $\left|U_{1}\right| \geqslant 3$ or $\left|U_{2}\right| \geqslant 3$. If $\left|U_{2}\right| \geqslant 3$, then $U_{0}=\left\{g_{1}-3 d, g_{1}-2 d, g_{1}-d\right\} \subset U_{2}$ and $U_{0}+x \subset S_{1}$, so $|(S+x) \backslash S|=|(S-x) \backslash S|=\left|(S-x) \cap U_{1}\right|+\left|(S-x) \cap U_{2}\right| \geqslant\left|U_{0}\right|=3$. The proof of the case $\left|U_{1}\right| \geqslant 3$ is similar.

Subcase 4.1.2: If $\left|S_{1}\right|<k$, let $H_{1}=\left\{g_{1}, g_{1}+k d, g_{1}+2 k d\right\}, H_{2}=H_{1}+d$ and $H_{3}=H_{1}+2 d$. Obviously, each of the 3 disjoint subsets of $C_{n}$ has 3 elements. Now we first prove that $H_{i} \not \subset S, i=1,2,3$. If $g_{1}+(i-1+k) d \in S$, then $g_{1}+(i-1+k) d \in S_{2}$ since $\left|S_{1}\right|<k$, and so $g_{1}+(i-1+2 k) d \notin S_{2}$. Notice that $g_{1}+(i-1+2 k) d \notin S_{1}$, otherwise, we would have $g_{1}+(i-1+2 k) d==g_{1}+m d$ for some integer $m, m \in\left[0,\left|S_{1}\right|-1\right]$, and so $2 k+2 \geqslant n=2 r$, which is impossible. It follows that $g_{1}+(i-1+2 k) d \notin S$. Since $g_{1}+(i-1) d \in S_{1}, i=1,2,3$, so each of $H_{i} \not \subset S, i=1,2,3$ contributes at least one element to $(S+x) \backslash S$. Hence $|(S+x) \backslash S| \geqslant 3$. 
Case 4.2: $k=2$. Since $\left|S_{1}\right| \geqslant\left|S_{2}\right|$ and $|S| \geqslant 5$, we have $\left|S_{1}\right| \geqslant 3$. Note that $\left|U_{1}\right|+\left|U_{2}\right|=n-|S| \geqslant 5$, so $\left|U_{1}\right| \geqslant 3$ or $\left|U_{2}\right| \geqslant 3$.

Subcase 4.2.1: If $\left|U_{2}\right| \geqslant 3$, then $\left(S_{1}-x\right) \backslash S=\left\{g_{1}-2 d, g_{1}-d\right\}$, and so $\left|\left(S_{1}-x\right) \backslash S\right|=$ 2. If $S_{2}-x \not \subset S$ then $|(S+x) \backslash S|=\left|\left(S_{1}-x\right) \backslash S\right|+\left|\left(S_{2}-x\right) \backslash S\right| \geqslant 3$. So we may assume $S_{2}-x \subset S$. If $\left|S_{2}\right| \geqslant 2$, then $g_{2}+d \in S_{2}$ and $g_{2}+d-2 d \notin S$, a contradiction. Hence $S_{2}=\left\{g_{2}\right\}$, and $g_{2}-2 d \notin S_{2}$ implies that $g_{2}-2 d \in S_{1}$. Since $S$ is not an arithmetic progression of difference $d, S$ must have the form of case (b).

Subcase 4.2.2: If $\left|U_{1}\right| \geqslant 3$, it is similar to the Subcase 4.2.1.

Case 4.3: $k=r-1$.

Subcase 4.3.1: $\left|S_{1}\right| \geqslant k$. By a similar argument as in Subcase 4.1 .1 we have that $|(S+x) \backslash S| \geqslant 3$

Subcase 4.3.2: $\left|S_{1}\right|<k$. Obviously, both $H_{1}=\left\{g_{1}, g_{1}+x, g_{1}+2 x\right\}$ and $H_{2}=H_{1}+d$ have 3 elements. By the same argument as in Subcase 4.1.2, we derive $H_{i} \not \subset S, i=1,2$ and $|(S+x) \backslash S| \geqslant 2$. To get the equality, we must have $\left\{g_{1}+2 d, g_{1}+3 d, \ldots, g_{1}+t_{1} d\right\}+x \subset S_{2}$, then $\left|S_{1}\right| \geqslant\left|S_{2}\right| \geqslant\left|S_{1}\right|-2$. It is just the case (c), which completes the proof of this case.

5. Let $S_{1}=\left\{g_{1}, g_{1}+d, \ldots, g_{1}+t_{1} d\right\}, S_{2}=\left\{g_{2}, g_{2}+d, \ldots, g_{2}+t_{2} d\right\}, S_{3}=\left\{g_{3}, g_{3}+\right.$ $\left.d, \ldots, g_{3}+t_{3} d\right\}$ and let $U_{1}=\left\{g_{1}+t_{1} d+d, g_{1}+t_{1} d+2 d, \ldots, g_{2}-d\right\}, U_{2}=\left\{g_{2}+t_{2} d+\right.$ $\left.d, g_{2}+t_{2} d+2 d, \ldots, g_{3}-d\right\}, U_{3}=\left\{g_{3}+t_{3} d+d, g_{3}+t_{3} d+2 d, \ldots, g_{1}-d\right\}$. Without loss of generality, we may assume that $S_{1}$ has the maximal length. Since $8 \leqslant|S| \leqslant n-7$, then $\left|S_{1}\right| \geqslant 3$. If $\left|U_{1}\right| \geqslant 2$ or $\left|U_{3}\right| \geqslant 2$, then it is easy to verify that $\left|\left(S_{1}+3 d\right) \backslash S\right| \geqslant 2$ or $\left|\left(S_{1}-3 d\right) \backslash S\right| \geqslant 2$, so both of them imply the result. Now we assume that $\left|U_{1}\right|=\left|U_{3}\right|=1$, then $\left|U_{2}\right| \geqslant 5$, and so $\left|\left(S_{2}+3 d\right) \backslash S\right| \geqslant 1$ and $\left|\left(S_{1}+3 d\right) \backslash S\right| \geqslant 1$, the result follows. This completes the proof of Lemma 5.2.

Now, we give some remarks about Lemma 5.2:

1. The equality of part 1 holds for all abelian groups $G$ and any element $x \in G$.

2. In part 2 of the Lemma, if $2 \leqslant|S| \leqslant n-2$, then $|(S+x) \backslash S|=1$ if and only if $x= \pm d$.

3. In part 4 of the Lemma, case (b) and case (c) do not hold simultaneously.

\section{Proof of the Theorem 1.1}

Proof. Let $X \subset G$ be a zero-sum free subset with $|X|=7$, and let $S=\sum(X)$. Without loss of generality, we may assume $G=\langle X\rangle$ and $|S| \leqslant 23$ for the contrary. By Lemmas 3.3 and 3.4, we may assume $r(G) \geqslant 2$ and all elements of $X$ have order greater than 2 . By Lemmas 3.1 and $3.2, f(X) \geqslant f(X \backslash\{x\})+f(x) \geqslant 19+1=20$ where $x \in X$, then we have $|G| \geqslant f(X)+1 \geqslant 21$. If there is an element $x \in X$ such that $|(S-x) \backslash S| \geqslant 5$, since $\sum(X \backslash\{x\}) \subset(S-x) \cap S$, we have that $|S| \geqslant f(X \backslash\{x\})+|(S-x) \backslash S| \geqslant f(X \backslash\{x\})+5 \geqslant 24$ by Lemma 3.2. Hence we may assume that $|(S-x) \backslash S| \leqslant 4$ for all $x \in X$. So, to

sum up, we may assume that $20 \leqslant|S| \leqslant 23,|G| \geqslant 21,<X>=G, r(G) \geqslant 2$ and $\operatorname{ord}(x)>2,|(S-x) \backslash S| \leqslant 4$ for all $x \in X$. The proof is divided to the following six cases. 
Case 1: $r(G) \geqslant 3$ and $|G| \geqslant 29$.

Since $|S| \leqslant 23$, by Lemma 4.6 , there is an element $x_{1} \in X$ such that $\left\langle X \backslash\left\{x_{1}\right\}\right\rangle=G$ and $\left|\sum\left(X \backslash\left\{x_{1}\right\}\right)\right| \leqslant|S|-4 \leqslant 19$. Now we apply Lemma 4.6 repeatedly, we will obtain $x_{2} \in X \backslash\left\{x_{1}\right\}, x_{3} \in X \backslash\left\{x_{1}, x_{2}\right\}$ such that $\left\langle X \backslash\left\{x_{1}, x_{2}\right\}\right\rangle=G,\left\langle X \backslash\left\{x_{1}, x_{2}, x_{3}\right\}\right\rangle=G$ and $f\left(X \backslash\left\{x_{1}, x_{2}\right\}\right) \leqslant f\left(X \backslash\left\{x_{1}\right\}\right)-4 \leqslant 15, f\left(X \backslash\left\{x_{1}, x_{2}, x_{3}\right\}\right) \leqslant f\left(X \backslash\left\{x_{1}, x_{2}\right\}\right)-4 \leqslant 11$. But we have that $\left|\sum\left(X \backslash\left\{x_{1}, x_{2}, x_{3}\right\}\right)\right| \geqslant 12$ by Lemma 5.1 , a contradiction.

Case 2: $G \cong C_{n} \oplus C_{n r}, n \geqslant 5$ and $|G| \geqslant 40$

Subcase 2.1: There is an element $x_{0} \in X$ such that ord $\left(x_{0}\right) \geqslant 5$. Let $H=\left\langle x_{0}\right\rangle$, then $|H| \geqslant 5$ and $|G / H| \geqslant 5$. Since $|(S-x) \backslash S| \leqslant 4$ for all $x \in X$, it follows from Lemma 4.4 that $\min \{|S|,|G \backslash S|\} \leqslant 4^{2}$. Notice that $|S| \geqslant 20$, then $|G \backslash S| \leqslant 16$ and $|S| \geqslant|G|-16 \geqslant 24$, a contradiction.

Subcase 2.2: If $\operatorname{ord}(x)<5$ for all $x \in X$, then $\operatorname{ord}(x) \in\{3,4\}$ for all $x \in X$.

We can choose 2 elements $x_{0}, x_{1} \in X$ such that $\operatorname{ord}\left(x_{0}\right)=3$ and $\operatorname{ord}\left(x_{1}\right)=4$. The choice is possible since otherwise we would have $\operatorname{ord}(x)=3$ for all $x \in X$ or $\operatorname{ord}(x)=4$ for all $x \in X$. Note that $r(G)=2$, so $G \cong C_{3} \oplus C_{3}, G \cong C_{4} \oplus C_{4}$ or $G \cong C_{2} \oplus C_{4}$, which contradicts $|G| \geqslant 40$. Let $H=\left\langle x_{0}, x_{1}\right\rangle$, then a similar discussion as in Subcase 2.1 will lead to a contradiction again.

Case 3: $\quad G \cong C_{4} \oplus C_{4 r}$ and $|G| \geqslant 40$.

Subcase 3.1: There is an element $x_{0} \in X$ such that $5 \leqslant \operatorname{ord}\left(x_{0}\right)<4 r$. Let $H=\left\langle x_{0}\right\rangle$, then the remaining discussion is similar to Subcase 2.1.

Subcase 3.2: $\operatorname{ord}(x) \in\{3,4,4 r\}$ for all $x \in X$.

We first prove the following 2 claims.

Claim 1: There is an element $x_{0} \in X$ such that ord $\left(x_{0}\right)=4 r$.

Proof of Claim 1: Since $f(6) \geqslant 19>\left|C_{4} \oplus C_{4}\right|$, then there is at most 5 elements of order 4 in $X$. Notice that there is at most 1 element of order 3 in $X$ and $|X|=7$, then Claim 1 follows.

Claim 2: Let $H=\left\langle x_{0}\right\rangle, x_{0} \in X$ and ord $\left(x_{0}\right)=4 r$, then $H \cap X=\left\{x_{0}\right\}$.

Proof of Claim 2: Let $a_{i}+H, a_{i} \in G / H, i=0,1,2,3$ denote the 4 cosets of $H$ in $G$. Let $S_{i}=\left(H+a_{i}\right) \cap S$ and define a function $f: G / H \rightarrow N$ by $f\left(a_{i}\right)=\left|S_{i}\right|$, then $\max (f)-\min (f) \leqslant 4$ by Lemma 4.3 .

Notice that $20 \leqslant|S| \leqslant 23$, so $2 \leqslant f\left(a_{i}\right) \leqslant|H|-2$ for all $i \in[0,3]$, and hence $\left|\left(S_{i}-x_{0}\right) \backslash S_{i}\right| \geqslant 1$ for all $i \in[0,3]$. Since $\left|\left(S-x_{0}\right) \backslash S\right| \leqslant 4$, it follows that each $S_{i}$ is an arithmetic progression of difference $x_{0}$. If there is another $x_{1} \in X \cap H$, say, $x_{1}=$ $k x_{0}, 2 \leqslant k \leqslant 4 r-2$, since $S_{i}, i \in[0,3]$ are arithmetic progressions of difference $x_{0}$ and $2 \leqslant\left|S_{i}\right| \leqslant|H|-2$, then $\left|\left(S_{i}-x_{1}\right) \backslash S_{i}\right| \geqslant 1$ and $\left|\left(S-x_{1}\right) \backslash S\right| \leqslant 4$ imply $\left|\left(S_{i}-x_{1}\right) \backslash S_{i}\right|=1$ and each $S_{i}, i \in[0,3]$ is an arithmetic progression of difference $x_{1}$. Hence $x_{1}= \pm x_{0}$ by Lemma 5.2, a contradiction. So Claim 2 holds.

Since each element of order 3 is contained in a cyclic subgroup of order $4 r$, by Claim 2, we have $\operatorname{ord}(x) \neq 3$ for any $x \in X$. Let $X=Y \cup Z$, where $Y$ consists of elements of order 4 and $Z$ consists of elements of order $4 r$, then $|Y| \leqslant 5$ by the proof of Claim 1 . Let $b \in X$ be an element with $\operatorname{ord}(b)=4 r$, choose $a \in G$ such that $G=\langle a\rangle \oplus\langle b\rangle$ and $\operatorname{ord}(a)=4$. Let $G_{0}=\langle a, r b\rangle$. Obviously, $Y \subset G_{0}$ and $Z \cap G_{0}=\emptyset$.

Subcase 3.2.1: If $2 \mid r$, then there are only 4 cyclic subgroups of order $4 r$ : $\langle b\rangle$, 
$\langle a+b\rangle,\langle-a+b\rangle$ and $\langle 2 a+b\rangle$. By Claim 2, a subgroup of order $4 r$ contributes at most 1 element of order $4 r$ to $X$, so $|Z| \leqslant 4$. It is easy to see that every element of order $4 r$ is of the form $k a+t b, \operatorname{gcd}(t, r)=1$.

If $|Y|=5$, then $S \supset \sum(Y) \uplus\{b\} \uplus\left(b+\sum(Y)\right)$ is a disjoint union and $|S| \geqslant 2\left|\sum(Y)\right|+$ $1 \geqslant 2 \times 13+1=27$ by Lemma 3.2 .

If $|Y|=4$, we let $Z=\left\{k_{1} a+t_{1} b, k_{2} a+t_{2} b, k_{3} a+t_{3} b\right\}, \operatorname{gcd}\left(t_{1} t_{2} t_{3}, r\right)=1$. If $\mid \sum\left(t_{1}, t_{2}, t_{3}\right)$ $(\bmod r) \backslash\{0\} \mid \geqslant 2$, say, $l_{1}, l_{2} \in \sum\left(t_{1}, t_{2}, t_{3}\right)(\bmod 2 r), 0 \not \equiv l_{1}, l_{2}(\bmod r), l_{1} \not \equiv l_{2}$ $(\bmod r)$, then $S \supseteq \sum(Y) \uplus\left(m_{1} a+l_{1} b+\sum(Y)\right) \uplus\left(m_{2} a+l_{2} b+\sum(Y)\right)$ and hence $|S| \geqslant 3\left|\sum(Y)\right| \geqslant 3 \times 8=24$ by Lemma 3.1. If $\left|\sum\left(t_{1}, t_{2}, t_{3}\right) \quad(\bmod r) \backslash\{0\}\right|<2$, then $t_{1} \equiv t_{2} \equiv t_{3} \quad(\bmod r)$ and $r=2$ since $\operatorname{gcd}\left(t_{1} t_{2} t_{3}, r\right)=1$ and $2 \mid r$, which contradicts $|G| \geqslant 40$.

If $|Y|=3$, we let $Z=\left\{k_{1} a+t_{1} b, \ldots, k_{4} a+t_{4} b\right\}, \operatorname{gcd}\left(t_{1} t_{2} t_{3} t_{4}, r\right)=1$. If $\mid \sum\left(t_{1}, t_{2}, t_{3}, t_{4}\right)$ $(\bmod r) \backslash\{0\} \mid \geqslant 3$, then similarly, we have $|S| \geqslant 4\left|\sum(Y)\right| \geqslant 4 \times 6=24$ by Lemma 3.1. If $\left|\sum\left(t_{1}, t_{2}, t_{3}, t_{4}\right) \quad(\bmod r) \backslash\{0\}\right| \leqslant 2$, then a similar discussion as in the case $|Y|=4$ shows that $r=2$ since $\operatorname{gcd}\left(t_{1} t_{2} t_{3} t_{4}, r\right)=1$ and $2 \mid r$, which also contradicts $|G| \geqslant 40$.

Subcase 3.2.2: If $2 \not \supset$, then there are precisely 6 cyclic subgroups of order $4 r$ : $\langle b\rangle,\langle a+b\rangle,\langle a+2 b\rangle,\langle-a+b\rangle,\langle a+4 b\rangle$ and $\langle 2 a+b\rangle$. Notice that any element of order 4 is contained in one of the 6 subgroups. By the Pigeonhole Principle, there is some subgroup $H$ which contributes at least 2 elements to $X$. By Claim 2, this subgroup $H$ contributes only elements of order 4 . However, 2 elements of order 4 in $H$ leads to a contradiction since $H$ has precisely two elements of order four: $r x,-r x$, where $x$ is a generator of $H$.

Case 4: $\quad G \cong C_{3} \oplus C_{3 r}$ and $|G| \geqslant 40$.

Subcase 4.1: There is an element $x_{0} \in X$ such that $5 \leqslant \operatorname{ord}\left(x_{0}\right)<3 r$, or two elements $x_{1}, x_{2} \in X$ with $\operatorname{ord}\left(x_{i}\right) \in\{3,4\}, i=1,2$. Let $H=\left\langle x_{0}\right\rangle$ or $\left\langle x_{1}, x_{2}\right\rangle$, $G_{1}=G / H$. Then both $H$ and $G / H$ have at least 5 elements. The remaining discussion is similar to Subcase 2.1.

Claim 3: Let $H=\left\langle x_{0}\right\rangle, x_{0} \in X$ and ord $\left(x_{0}\right)=3 r$, then $H \cap X=\left\{x_{0}\right\}$.

Proof of Claim 3: Let $H+a_{i}, a_{i} \in G / H, i=0,1,2$ denote the 3 cosets of $H$ in $G$. Let $S_{i}=\left(H+a_{i}\right) \cap S$ and define a function $f: G / H \rightarrow N$ by $f\left(a_{i}\right)=\left|S_{i}\right|$, then $\max (f)-\min (f) \leqslant 4$ by Lemma 4.3 .

Notice that $20 \leqslant|S| \leqslant 23$, so $4 \leqslant f\left(a_{i}\right) \leqslant|H|-3$ for any $i \in[0,2]$. Since $\mid\left(S_{i}-\right.$ $\left.x_{0}\right) \backslash S_{i} \mid \geqslant 1$ and $\left|\left(S-x_{0}\right) \backslash S\right| \leqslant 4$, without loss of generality, we may assume that $S_{0}$ and $S_{1}$ are arithmetic progressions of difference $x_{0}$. If there is another $x_{1} \in X \cap H$, then by Lemma $5.2\left|\left(S_{0}+x_{1}\right) \backslash S_{0}\right| \geqslant 2,\left|\left(S_{1}+x_{1}\right) \backslash S_{1}\right| \geqslant 2$ and $\left|\left(S_{2}+x_{1}\right) \backslash S_{2}\right| \geqslant 1$ imply that $\left|\left(S+x_{1}\right) \backslash S\right| \geqslant 5$, a contradiction, so the claim holds.

Since all the elements of order 4 are included in the cyclic subgroup of order $3 r$, by Claim 3 above, we have ord $(x) \neq 4$ for any $x \in X$. Let $X=Y \cup Z$, where $Y$ consists of elements of order 3 and $Z$ consists of elements of order $3 r$, then $|Y| \leqslant 1$ by Subcase 4.1. Choose $a, b \in G$ such that $G=\langle a\rangle \oplus\langle b\rangle$, and ord $(b)=3 r$.

If $3 \mid r$, then there are only 3 cyclic subgroups of order $3 r:\langle b\rangle,\langle a+b\rangle$ and $\langle-a+b\rangle$. If $3 \not \gamma$, then there are precisely 5 cyclic subgroups of order $3 r:\langle b\rangle,\langle a+b\rangle,\langle-a+b\rangle$, $\langle a-3 b\rangle$ and $\langle a+3 b\rangle$. By Claim 4, every subgroup of order $3 r$ will contribute at most 1 element of order $3 r$ to $X$, so $|Z| \leqslant 5$. It follows that $|Y|+|Z| \leqslant 6$, a contradiction. 
Case 5: $\quad G \cong C_{2} \oplus C_{2 r}$ and $|G| \geqslant 40$.

Subcase 5.1: There is an element $x_{0} \in X$ such that $5 \leqslant \operatorname{ord}\left(x_{0}\right)<r$. Let $H=\left\langle x_{0}\right\rangle$, then the discussion is similar to Subcase 2.1 .

Subcase 5.2: For any $x \in X$, ord $(x) \in\{3,4, r, 2 r\}$.

Choose $a, b \in G$ such that $G=\langle a\rangle \oplus\langle b\rangle, \operatorname{ord}(a)=2$ and $\operatorname{ord}(b)=2 r$. Let $X=$ $X_{3} \cup X_{4} \cup X_{r} \cup X_{2 r}$, where $X_{i}, i \in\{3,4, r, 2 r\}$ consists of elements of order $i$. Let $G_{0}=<a, 2 b>\nsubseteq \subseteq G$, it is easy to verify that $x \in G_{0}$ for any $x \in G$ with $\operatorname{ord}(x) \in\{3, r\}$. There are at most 2 cyclic subgroups of order 4 in $G:\left\langle\frac{r}{2} b\right\rangle$ and $\left\langle a+\frac{r}{2} b\right\rangle$, each contributes at most 1 element of order 4 to $X$, so $\left|X_{4}\right| \leqslant 2$. If $\left|X_{4}\right|=2$, let $H=<x_{4}>$, then a similar discussion as in Subcase 2.1 leads to a contradiction. Now again we need 3 claims.

Claim 4: $X_{2 r} \neq \emptyset$

Proof of Claim 4: Suppose that $X_{2 r}=\emptyset$. If there is an element $x_{4} \in X_{4}$ such that $x_{4} \notin G_{0}$, then $S \supset \sum\left(X_{3} \cup X_{r}\right) \uplus\left\{x_{4}\right\} \uplus\left(x_{4}+\sum\left(X_{3} \cup X_{r}\right)\right)$ is a disjoint union and hence $|S| \geqslant 2\left|\sum\left(X_{3} \cup X_{r}\right)\right|+1 \geqslant 2 \times 13+1=27$, a contradiction. It follows that either $X_{4}=\emptyset$ or $X_{4} \subset G_{0}$, which implies $G=<X>\subset G_{0} \nsubseteq \subseteq$, a contradiction again. So the Claim 4 holds.

Claim 5: Let $x_{2 r} \in X_{2 r}$ and $H=<x_{2 r}>$, if there is another $y \in H \cap X$, then $y= \pm 2 x_{2 r}$ or $y= \pm(r-1) x_{2 r}$. Furthermore, $|H \cap X| \leqslant 2$.

Proof of Claim 5: Let $H+a_{i}, a_{i} \in G / H, i=0,1$ denote the 2 cosets of $H$ in $G$. Let $S_{i}=\left(H+a_{i}\right) \cap S$ and define a function $f: G / H \rightarrow N$ by $f\left(a_{i}\right)=\left|S_{i}\right|$, then $\max (f)-\min (f) \leqslant 4$ by Lemma 4.3

Notice that $20 \leqslant|S| \leqslant 23$, so $8 \leqslant f\left(a_{i}\right) \leqslant|H|-7$ for any $i \in[0,1]$. Since $\mid\left(S_{i}-\right.$ $\left.x_{2 r}\right) \backslash S_{i} \mid \geqslant 1$ and $\left|\left(S-x_{2 r}\right) \backslash S\right| \leqslant 4$, then we have that $S_{i}, i=0,1$ is the union of at most 3 arithmetic progressions of difference $x_{2 r}$.

If there is some $S_{i}$ which is an arithmetic progression of difference $x_{2 r}$, without loss of generality, we may assume that is $S_{0}$. If $y \neq \pm 2 x_{2 r}$, since $y \neq \pm x_{2 r}$, we have $y= \pm 3 x_{2 r}$ or $y \in<x_{2 r}>\backslash\left\{ \pm x_{2 r}, \pm 2 x_{2 r}, \pm 3 x_{3 r}\right\}$. If $y= \pm 3 x_{2 r}$, then $\left|\left(S_{0}+y\right) \backslash S\right|=3$ and $\left|\left(S_{1}+y\right) \backslash S\right| \geqslant$ 2 by Lemma 5.2.5. . If $y= \pm 4 x_{2 r}$, then $\left|\left(S_{0}+y\right) \backslash S\right|=4$ and $\left|\left(S_{1}+y\right) \backslash S\right| \geqslant 1$. Otherwise, $\left|\left(S_{0}+y\right) \backslash S\right| \geqslant 5$. It follows that $|(S+y) \backslash S| \geqslant 5$, a contradiction.

If there is no $S_{i}$ which is an arithmetic progression, then we have that $S_{i}, i=0,1$ both are the unions of 2 arithmetic progressions of difference $x_{2 r}$. Since $y \neq \pm x_{2 r}$, we have $\left|\left(S_{i}+y\right) \backslash S_{i}\right| \geqslant 3$, or $y= \pm 2 x_{2 r}$, or $y= \pm(r-1) x_{2 r}$ by Lemma 5.2.4, and the claim holds.

By the hypothesis, we have that $\pm 2 x_{2 r}$ has order $r$ and $\pm(r-1) x_{2 r}$ has order $2 r$ if $2 \mid r$ and order $r$ if $2 \not \supset r$.

Claim 6: Let $x_{r} \in X_{r}$ and $K=<x_{r}>$, then $X_{r} \cap K=\left\{x_{r}\right\}$.

Proof of Claim 6: By a similar argument as in the proof of Claim 2, we obtain Claim 6.

Since all elements of order 3 are contained in cyclic subgroups of order $2 r$, by Claim 5 and $r \neq 3$, we have $X_{3}=\emptyset$.

Subcase 5.2.1: If $2 \not \backslash r$, then there are precisely 3 cyclic subgroups of order $2 r$ : $\langle b\rangle,\langle a+b\rangle$ and $\langle a+2 b\rangle$. In this subcase, $X_{4}=\emptyset$. Claim 5 and the discussion after imply that each subgroup of order $2 r$ contributes at most 1 element of order $2 r$, so $\left|X_{2 r}\right| \leqslant 3$ and $\left|X_{r}\right|=|X|-\left|X_{2 r}\right| \geqslant 4$. Notice that $X_{r} \subset G_{0}$ and $<a+2 b>\subset G_{0}$, then 
$\left|X_{2 r} \backslash G_{0}\right| \leqslant 2$. If $\left|X_{2 r} \backslash G_{0}\right|=0$, then $X \subset G_{0}$, a contradiction. Therefore we can choose $x_{2 r} \in X_{2 r} \backslash G_{0}$. Now $S \supset \sum\left(G_{0} \cap X\right) \oplus\left\{x_{2 r}\right\} \oplus\left(x_{2 r}+\sum\left(G_{0} \cap X\right)\right)$ and $\left|G_{0} \cap X\right| \geqslant 5$ imply that $|S| \geqslant 2 \times 13+1=27$, a contradiction.

Subcase 5.2.2: If $2 \mid r$, then there are only 2 cyclic subgroups of order $2 r: H_{1}=<$ $b>$ and $H_{2}=\langle a+b\rangle$. Let $H$ be a cyclic subgroup of order $2 r$, if $X_{2 r} \cap H \neq \emptyset$, then $|X \cap H| \leqslant 2$ by Claim 5 ; if $X_{2 r} \cap H=\emptyset$, then $\left|X_{r} \cap H\right| \leqslant 1$ by Claim 6 , and so $|X \cap H| \leqslant 2$ since $\left|X_{4} \cap H\right| \leqslant 1$. It follows that $H$ contributes at most 2 elements to $X$.

If $4 \not \gamma$, then all the elements of order 4 are contained in the subset $H_{1} \cup H_{2}$. Note that $G$ has precisely 3 cyclic subgroups of order $r:\langle 2 b\rangle c\langle b\rangle,\langle a+2 b\rangle$ and $\langle a+4 b\rangle$. A cyclic subgroups of order $r$ contributes at most 1 element to $X$ by Claim 6 . It follows that $|X| \leqslant 2 \times 2+1+1=6<7$, a contradiction.

If $4 \mid r$, let $r_{0}=r / 2$. Then $G$ has precisely 2 cyclic subgroups of order $\left.4:\left\langle r_{0} b\right\rangle c<b\right\rangle$ and $\left\langle a+r_{0} b\right\rangle$, and 2 cyclic subgroups of order $\left.r:\langle 2 b\rangle c<b\right\rangle$ and $\langle a+2 b\rangle$. A cyclic subgroups of order 4 or $r$ contributes at most 1 element to $X$ by Claim 6 . It follows that $|X| \leqslant 2 \times 2+1+1=6<7$, a contradiction again.

Case 6: $G$ with small order.

Since $|G| \geqslant 21$ and $r(G) \geqslant 2$, the left cases of $G$ are of the following forms: $C_{3} \oplus C_{3} \oplus C_{3}$, $C_{2} \oplus C_{2} \oplus C_{6}, C_{6} \oplus C_{6}, C_{5} \oplus C_{5}, C_{4} \oplus C_{8}, C_{3} \oplus C_{9}, C_{3} \oplus C_{12}, C_{2} \oplus C_{12}, C_{2} \oplus C_{14}, C_{2} \oplus C_{16}$ and $C_{2} \oplus C_{18}$.

To begin with, since $D\left(C_{3} \oplus C_{3} \oplus C_{3}\right)=7$, we have $f\left(C_{3} \oplus C_{3} \oplus C_{3}, 7\right)=26$.

The remaining cases are computed with a $\mathrm{C}++$ program. With the help of a computer, we obtain the following values:

$\begin{array}{ll}\text { Result } & \text { Running Time(sec) } \\ \text { ol }\left(C_{2} \oplus C_{2} \oplus C_{6}\right)=6 & 7.6 \\ f\left(C_{6} \oplus C_{6}, 7\right)=29 & 98 \\ \text { ol }\left(C_{5} \oplus C_{5}\right)=6 & 1.0 \\ f\left(C_{4} \oplus C_{8}, 7\right)=27 & 26 \\ \text { ol }\left(C_{3} \oplus C_{9}\right)=6 & 3.9 \\ f\left(C_{3} \oplus C_{12}, 7\right)=27 & 190 \\ \text { ol }\left(C_{2} \oplus C_{12}\right)=6 & 1.2 \\ f\left(C_{2} \oplus C_{14}, 7\right)=25 & 8.7 \\ f\left(C_{2} \oplus C_{16}, 7\right)=25 & 51 \\ f\left(C_{2} \oplus C_{18}, 7\right)=25 & 285\end{array}$

This completes the proof.

\section{References}

[1] R.B. Eggleton and P. Erdős, Two combinatorial problems in group theory, Acta Arith. 21(1972), 111-116.

[2] J.E. Olson, Sums of sets of group elements, Acta Arith. 28 (1975), 147-156. 
[3] W. Gao, Y. Li, J. Peng and F. Sun, Subsums of a zero-sum free subset of an abelian group, The Electronic Journal of Combinatorics, 15(2008), \#R116.

[4] W. Gao, Y. Li, J. Peng and F. Sun, On subsequences sums of a zero-sum free sequence II, The Electronic Journal of Combinatorics, 15(2008), \#R117.

[5] G. Bhowmik, I. Halupczok, and J.-C. Schlage-Puchta, Zero-sum free sequences with small sum-set, eprint arXiv:0804.0313.

[6] A. Geroldinger and F. Halter-Koch, Non-Unique Factorizations. Algebraic, Combinatorial and Analytic Theory, Pure and Applied Mathematics, Vol. 278, Chapman \& Hall/CRC, 2006.

[7] A. Pixton, Sequences with small subsums sets, J. Number Theory 129(2009), 806817. 